\title{
Surgical outcomes of the endonasal endoscopic approach within a standardized management protocol for repair of spontaneous cerebrospinal fluid rhinorrhea
}

\author{
Daniel C. Kreatsoulas, MD, ${ }^{1}$ Varun S. Shah, BS, ${ }^{2}$ Bradley A. Otto, MD, ${ }^{3}$ Ricardo L. Carrau, MD, ${ }^{3}$ \\ Daniel M. Prevedello, MD, ${ }^{1,3}$ and Douglas A. Hardesty, MD ${ }^{1,3}$
}

Departments of ${ }^{1}$ Neurological Surgery and ${ }^{3}$ Otolaryngology-Head and Neck Surgery, The Ohio State University Wexner Medical Center; and ${ }^{2}$ The Ohio State University College of Medicine, Columbus, Ohio

\begin{abstract}
OBJECTIVE Spontaneous CSF leaks are rare, their diagnosis is often delayed, and they can precipitate meningitis. Craniotomy is the historical "gold standard" repair for these leaks. An endonasal endoscopic approach (EEA) offers potentially less invasiveness and lower surgical morbidity than a traditional craniotomy but must yield the same surgical success. A paucity of data exists studying EEA as the primary management for spontaneous CSF leaks.
\end{abstract}

METHODS The authors retrospectively reviewed patients undergoing spontaneous CSF rhinorrhea repair at their institution from July 2010 to August 2018. Standardized management includes EEA as first-line treatment, and lumbar puncture (LP) performed 24-48 hours postoperatively. If opening pressure on LP is elevated, CSF diversion or acetazolamide therapy is used as needed. Perioperative lumbar drains are not used.

RESULTS Of 46 patients identified, the most common CSF rhinorrhea etiology was encephalocele $(28 / 46,60.9 \%)$, and the most common location was cribriform/ethmoid (26/46, 56.5\%). Forty-three patients underwent EEA alone, and 3 underwent a simultaneous EEA/craniotomy. The most common repair strategy was nasoseptal or other pedicled flaps (18/46, 39.1\%). Postoperatively, 15 patients (32.6\%) received CSF diversion due to elevated ICP, with BMI > $40 \mathrm{~kg} / \mathrm{m}^{2}$ being a significant risk factor (odds ratio 4.35, $p=0.033$ ) for postrepair shunt placement. Twelve patients received acetazolamide therapy for treatment of mildly elevated pressures. The average opening pressure of the shunted group was $36 \mathrm{~cm} \mathrm{H}_{2} \mathrm{O}$ and the average for the acetazolamide-only group was $26 \mathrm{~cm} \mathrm{H}_{2} \mathrm{O}$. Two patients underwent CSF leak repair revision, one because of progressive fungal sinusitis and the other because of recurrent CSF leak. The mean follow-up duration was 15 months.

CONCLUSIONS The paradigm of EEA repair of spontaneous CSF rhinorrhea with postoperative LP to identify undiagnosed idiopathic intracranial hypertension appears to be safe and effective. In the authors' cohort, morbid obesity was statistically associated with the need for postoperative CSF diversion. This has implications for future surgical treatment as obesity levels continue to rise worldwide.

https://thejns.org/doi/abs/10.3171/2019.12.JNS192891

KEYWORDS cerebrospinal fluid leak; endonasal endoscopic approach; EEA; CSF rhinorrhea; pituitary surgery

$\mathrm{S}$ PONTANEOUS skull base CSF leaks are rare pathologies that offer a diagnostic and therapeutic conundrum and may result in serious consequences such as meningitis. Elevated intracranial pressure (ICP), such as from idiopathic intracranial hypertension (IIH), is one common etiology of spontaneous CSF leaks. ${ }^{2}$ An increase in worldwide obesity may be increasing the incidence of $\mathrm{IIH}$ and subsequently leading to a higher incidence of spontaneous CSF leaks in the modern era. ${ }^{8}$ Other potential etiologies for spontaneous CSF rhinorrhea include trauma and prior infection; both presumably structurally weaken the anterior skull base.

Open craniotomy is the traditional gold standard for repair of sinonasal CSF leaks due to a wide view and relative ease of access to common areas and repair materials (such as a pericranial flap). Other strategies or adjuncts for treatment include medical CSF production reduction (i.e., acetazolamide therapy) or CSF diversion (placement of a

ABBREVIATIONS EEA = endonasal endoscopic approach; ICP = intracranial pressure; IIH = idiopathic intracranial hypertension; $L P=$ lumbar puncture; $O R=$ odds ratio. SUBMITTED October 24, 2019. ACCEPTED December 16, 2019.

INCLUDE WHEN CITING Published online February 28, 2020; DOI: 10.3171/2019.12.JNS192891. 
lumbar drain or CSF shunt) to reduce ICP. Perioperative lumbar drains have not been shown to significantly decrease the rate of CSF leak repair failure..$^{5,8}$

With the advent of improved repair techniques and the durability of pedicled mucosal flaps, an endonasal endoscopic approach (EEA) offers the possibility of no external incisions and potentially lower surgical morbidity than does a traditional craniotomy. Modern repair techniques for postoperative defects (i.e., surgical/iatrogenic CSF leaks from tumor resection) are durable and have a low failure rate. ${ }^{4}$ Several small cohorts demonstrate relatively good repair success rates of $80 \%-94 \%$ for spontaneous leaks via endoscopic techniques but have not addressed the management of underlying concurrent IIH. . $^{1,3,6,10,11,13}$ Our institution utilizes a standard protocol: EEA repair as primary treatment of the CSF leak, and standard postoperative measurement of ICP (if applicable based on etiology) to elucidate the possible need for permanent CSF diversion for $\mathrm{IIH}$, in addition to skull base repair. In this paper we aim to demonstrate the efficacy and durability of this approach for the treatment of spontaneous CSF rhinorrhea.

\section{Methods \\ Study Criteria}

The authors retrospectively reviewed patients diagnosed with spontaneous CSF leaks who underwent surgical repair at The Ohio State University Wexner Medical Center between July 2010 and August 2018. IRB approval was obtained prior to the study. Variables assessed included demographics, prior predisposing pathologies, prior intervention for CSF leak, BMI, and surgical techniques/ outcomes. The data were analyzed utilizing SPSS (version 25 , IBM Corp.), with binary regression models to arrive at odds ratios (ORs) for predictive factors.

\section{Treatment Paradigm}

All patients were managed utilizing a standardized paradigm. Patients with suspected CSF rhinorrhea undergo routine awake nasal endoscopy, and if there is an ambiguous finding, a sample of the rhinorrhea is sent to the laboratory for testing for beta- 2 transferrin. Thereafter, imaging of the potential CSF leak location via CT and MRI is performed. No preoperative lumbar puncture (LP) is performed, unless there is a concern for meningitis at presentation, or if it was performed prior to the patient being seen at our institution. Surgical repair via endoscopic endonasal surgery using a combined otolaryngological and neurosurgical approach is the first-line treatment. A variety of local vascularized mucosal flaps, free mucosal grafts, and artificial collagen dural matrix are utilized on a case-by-case basis. An example of pre- and postoperative imaging coupled with endoscopic images of a pedicled nasoseptal flap and middle turbinate graft can be seen in Fig. 1. Combined craniotomy simultaneous with EEA is rarely added per the discretion of the primary neurosurgeon. The patients are cared for in the intensive care unit if a craniotomy is performed, and in a step-down unit if only an EEA is performed. We do not routinely use lumbar drains in these patients. An LP is performed 24-48 hours postoperatively unless the patient has a compel- ling contraindication. If the opening pressure is significantly elevated (i.e., $>30 \mathrm{~cm} \mathrm{H}_{2} \mathrm{O}$ ), or if significant other factors are present (i.e., large defect, concern for patient compliance, very high BMI), a ventriculoperitoneal or lumboperitoneal shunt is placed during the same admission to reduce ICP. If the opening pressure is only mildly elevated, or if a patient does not wish to undergo shunt placement, acetazolamide therapy is used to medically manage mild IIH. Patients are followed postoperatively by neurosurgery and otolaryngology, with routine nasal care and debridement as needed. Patients are postoperatively referred to ophthalmology, with either an inpatient consultation or outpatient evaluation, if IIH has been diagnosed.

\section{Results}

\section{Patient Characteristics}

We identified 46 patients who underwent 49 total spontaneous CSF leak repair procedures during the study period (Table 1), with a mean postoperative follow-up duration of 15 months. The patient cohort was a majority female (n $=37,80.4 \%$ ) and obese (mean BMI $39.8 \mathrm{~kg} / \mathrm{m}^{2}$ ) cohort. Common presenting symptoms were isolated CSF rhinorrhea $(\mathrm{n}=22,47.8 \%)$, headache $(\mathrm{n}=16,34.8 \%)$, and rhinitis/postnasal drip $(n=4,8.7 \%)$. Three patients $(6.5 \%)$ presented with meningitis, 3 had a history of skull base injury from trauma (6.5\%), and 4 had a prior diagnosis of IIH $(8.7 \%)$. Of the 3 patients with prior remote trauma, 2 developed encephaloceles through suspected dehiscent areas of prior fracture in the skull base, while the third patent suffered from a skull base metastasis that led to erosion of the bone after chemotherapy treatment, and creation of a CSF fistula. Twelve patients (26.1\%) had a history of prior surgery at another facility for CSF leaks. Of these 12 patients with prior surgery, 10 underwent evaluation for increased ICP according to our standard protocol. For the 2 with a surgical history that deviated from our paradigm and did not undergo postoperative LP, one had a clear history of CSF leak from trauma and the other had an LP performed at a prior institution and was found to have high ICP, prompting the referral to our center. Thirty-one (67.4\%) of 46 patients had rhinorrhea samples that tested positive for beta- 2 transferrin through our institution or another laboratory. The other patients had direct visualization of an apparent leak through nasal endoscopy.

\section{Intraoperative Findings}

Intraoperative findings and surgical variables are described further in Table 2 . Three (6.5\%) of 46 patients had intrathecal fluorescein injections to help establish the site of CSF leakage. Use of fluorescein was only on a case-bycase basis, but not part of the standard protocol. The most common preoperative diagnosis for leak etiology was encephalocele $(28 / 46,60.9 \%)$. The most common location for a CSF leak was the cribriform/ethmoid region $(\mathrm{n}=$ $26,56.5 \%$ ), followed by the lateral recess of the sphenoid sinus $(\mathrm{n}=10,21.7 \%$; Table 1$)$. There were 2 patients in whom no obvious leak was identified intraoperatively: one patient had eroded bone in the lateral sphenoid sinus but was not actively leaking CSF during surgery. The sphenoid sinus was packed with fat graft as a repair. The other 

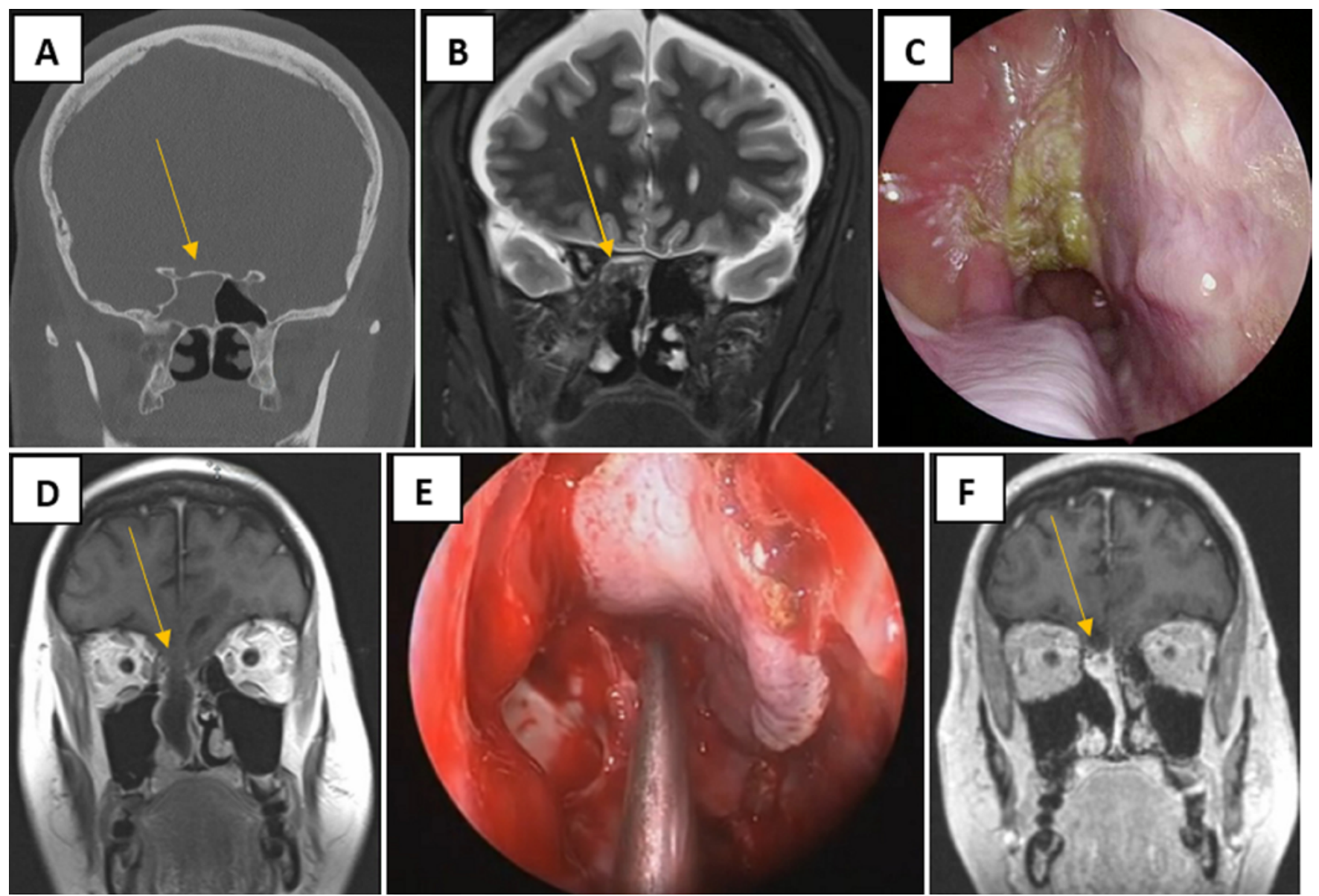

FIG. 1. A: Preoperative CT image of the sinuses in a patient with CSF rhinorrhea, demonstrating the sphenoid sinus filled with fluid and thinning of the bony roof (arrow). B: Postoperative T2-weighted MR image demonstrating interval packing of the sphenoid sinus with middle turbinate mucosal graft (arrow). C: Nasal endoscopy 6 months postoperatively, showing healed middle turbinate graft. D: Preoperative T1-weighted MR image with contrast, demonstrating meningoencephalocele protruding through the cribriform plate (arrow). E: Intraoperative endoscopic image showing placement of a pedicled nasoseptal flap over an operative defect. F: Postoperative T1-weighted MR image with contrast, demonstrating a well-healed nasoseptal pedicled flap (arrow) and interval reduction of meningoencephalocele. Figure is available in color online only.

patient had clear rhinorrhea that improved with acetazolamide treatment at an outside hospital; when that patient received surgery at our institution, no area of definitive CSF leak was found, and the sphenoid sinus was also packed with fat graft. Fifty-eight percent of the patients (n $=27$ ) had an identifiable encephalocele intraoperatively. Forty-three patients underwent EEA alone as definitive surgical management, and 3 required simultaneous EEA and craniotomy. Most procedures $(n=30,65.2 \%)$ reported minimal to $100 \mathrm{ml}$ of intraoperative blood loss. The most common repair strategy was nasoseptal or other pedicled flaps $(\mathrm{n}=18,39.1 \%)$. Turbinate mucosal grafting was used as repair in 10 patients $(21.7 \%)$, and abdominal fat grafting in 8 patients (17.4\%). A graphic representation of the repair types utilized can be found in Fig. 2. No lumbar drains were placed in any patients who were included in the protocol.

\section{Postoperative Course}

Surgical and medical complications were rare and are listed in Table 3. Two patients (4.4\%) underwent revision of their skull base repair during the follow-up period. One patient suffered a recurrent CSF leak during the surgical hospital admission due to repair failure. The second patient underwent several reoperations because of progressive fungal sinusitis that complicated an original head and neck cancer. Both patients were managed with repeat EEA surgery. One patient (2.2\%) suffered seizures both postoperatively and during follow-up. Two patients developed bacterial meningitis postoperatively (4.4\%). Five patients $(33 \%)$ who received CSF shunts required subsequent revision during clinical follow-up, although none had shunt explantation during the follow-up period. One patient developed chronic bilateral subdural hematomas during follow-up, related presumably to overdrainage after a lumboperitoneal shunt was placed. This patient was managed by increasing the pressure of the shunt valve. Two patients with cancer died during the follow-up period. Overall, only 2 patients required repair revision surgery for recurrent leaks during the follow-up period, leading to a $95.7 \%$ first-repair success rate. 
TABLE 1. Patient demographics $(n=46)$

\begin{tabular}{lc}
\hline \multicolumn{1}{c}{ Variable } & Value \\
\hline Mean age (range), yrs & $52.2(17-76)$ \\
\hline Sex, $\mathrm{n}(\%)$ & $9(19.6)$ \\
\hline Male & $37(80.4)$ \\
\hline Female & $39.8(20.8-64.7)$ \\
\hline Mean BMI (range), $\mathrm{kg} / \mathrm{m}^{2}$ & $3(6.5)$ \\
\hline History of trauma, $\mathrm{n}(\%)$ & $12(26.1)$ \\
\hline History of prior surgery for CSF leak, $\mathrm{n}(\%)$ & $3(6.5)$ \\
\hline History of prior medical treatments for IIH, $\mathrm{n}(\%)$ & $4(8.7)$ \\
\hline History of prior diagnosis of IIH, $\mathrm{n}(\%)$ & $26(56.5)$ \\
\hline CSF leak location, $\mathrm{n}(\%)$ & $10(21.7)$ \\
\hline Cribriform/ethmoid & $10(21.7)$ \\
\hline Sphenoid sinus lateral recess & \\
\hline Other & $22(47.8)$ \\
\hline Presenting symptoms, $\mathrm{n}(\%)$ & $3(6.5)$ \\
\hline CSF rhinorrhea only & $4(8.7)$ \\
\hline Meningitis & $16(34.8)$ \\
\hline Rhinitis/postnasal drip & $7(15.2)$ \\
\hline Migraines/headaches & $28(60.9)$ \\
\hline Other & $18(39.1)$ \\
\hline Imaging findings, $\mathrm{n}(\%)$ & \\
\hline Encephalocele & \\
\hline Other (bone defect only, sinonasal fluid, etc.) & \\
\hline * Other leak locations included maxillary sinus $(\mathrm{n}=2)$, clivus/posterior sella \\
skull base ( $\mathrm{n}=3$ ).
\end{tabular}

TABLE 2. Intraoperative findings

\begin{tabular}{lc}
\hline \multicolumn{1}{c}{ Variable } & Value (\%) \\
\hline Type of operation & $43(93.5)$ \\
\hline EEA alone & $3(6.5)$ \\
\hline EEA + craniotomy & $3(6.5)$ \\
\hline Utilization of fluorescein dye & $30(65.2)$ \\
\hline Blood loss & $9(19.6)$ \\
\hline Minimal to 100 ml & $7(15.2)$ \\
\hline$>100$ ml & $2(4.4)$ \\
\hline Not listed & $14(30.4)$ \\
\hline Pathology identified & $27(58.7)$ \\
\hline No obvious leak found & $3(6.5)$ \\
\hline Leak alone & $18(39.1)$ \\
\hline Leak + encephalocele & $10(21.7)$ \\
\hline Other & $8(17.4)$ \\
\hline Type of repair & $5(10.9)$ \\
\hline Nasoseptal or other pedicled flap & $4(8.7)$ \\
\hline Turbinate graft only & $1(2.2)$ \\
\hline Abdominal fat graft only &
\end{tabular}

$\mathrm{NA}=$ not available.

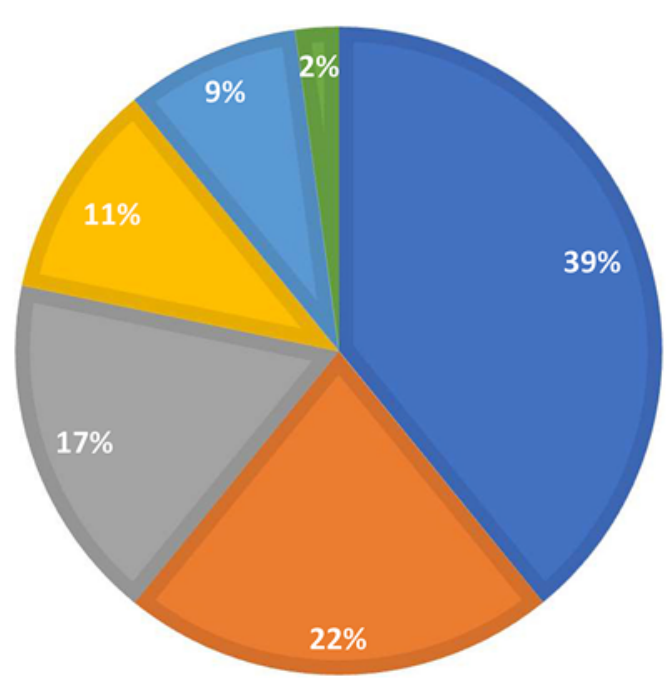

Nasoseptal or other pedicled flap

Turbinate graft only

Abdominal fat graft only

Combination repair

Free mucosal graft

N/A

FIG. 2. Representation of repair type by percentage of patients. N/A = not available. Figure is available in color online only

\section{Postoperative LP and Shunt Placement}

Postoperative LPs were performed for evaluation of CSF pressure on 34 (73.9\%) of the 46 patients at a median of 2 days after surgery (Table 4). Those who did not receive the postoperative $\mathrm{LP}$ fit into one of three catego-

\section{TABLE 3. Postoperative course}

\begin{tabular}{lc}
\hline \multicolumn{1}{c}{ Variable } & Value \\
\hline In-hospital complications, $\mathrm{n}(\%)$ & $2(4.4)$ \\
\hline Return to operating room for additional repair & $1(2.2)$ \\
\hline Seizures & $34(73.9)$ \\
\hline No. of patients undergoing LP, $\mathrm{n}(\%)$ & $15(32.6)$ \\
\hline No. of patients undergoing shunt procedures, $\mathrm{n}(\%)$ & \\
\hline Late-term complications, $\mathrm{n}(\%)$ & $1(2.2)$ \\
\hline Seizures & $2(4.4)$ \\
\hline Meningitis & $4(8.7)$ \\
\hline Shunt malfunction/misplacement & $1(2.2)$ \\
\hline Subdural hematoma & $2(4.4)$ \\
\hline Outcome at 1 mo postop, $\mathrm{n}(\%)$ & $41(89.1)$ \\
\hline Recurrent CSF leak & $1(2.2)$ \\
\hline No issues, flap healing well & $1(2.2)$ \\
\hline Surgical site infection & $1(2.2)$ \\
\hline Chronic sinusitis & $15(1-67)$ \\
\hline Repeat hospitalization
\end{tabular}


TABLE 4. Postoperative LP findings

\begin{tabular}{lc}
\hline \multicolumn{1}{c}{ Variable } & Value \\
\hline Patients undergoing LP (\%) & $34 / 46(73.9)$ \\
\hline Median postop days undergoing LP (range) & $2(0-3)$ \\
\hline LPs w/ ICP $>20 \mathrm{~cm} \mathrm{H}_{2} \mathrm{O}(\%)$ & $27 / 34(79.4)$ \\
\hline Mean ICP, $\mathrm{cm} \mathrm{H}_{2} \mathrm{O}$ & 36 \\
\hline Shunt group & 26 \\
\hline Nonshunt group & \\
\hline Types of shunts utilized, $\mathrm{n}(\%)$ & $6 / 15(40)$ \\
\hline Ventriculoperitoneal & $9 / 15(60)$ \\
\hline Lumboperitoneal & $4 / 15(26.7)$ \\
\hline Patients undergoing shunt revision (\%)
\end{tabular}

ries: 1) a clear etiology of leak not related to increased ICP $(\mathrm{n}=6,50 \%), 2)$ preoperative LP or diagnosis of IIH was already present $(\mathrm{n}=5,41.7 \%)$, or 3 ) the patient deferred $(\mathrm{n}=1,8.3 \%)$. Of the performed LPs, $27 / 34(79.4 \%)$ revealed an opening pressure $>20 \mathrm{~cm} \mathrm{H}_{2} \mathrm{O}$. Twelve $(44.4 \%)$ of these 27 patients were placed on acetazolamide therapy for mildly elevated pressures, and the other $15(55.6 \%)$ received permanent CSF diversion. None of the patients originally placed on acetazolamide therapy required escalation to CSF diversion; 2 of the 12 discontinued medication during follow-up after repeat LPs with normal opening pressure and no clinical signs of IIH. The average ICP of the shunted group was $36 \mathrm{~cm} \mathrm{H}_{2} \mathrm{O}$ and the average for the acetazolamide-only group was $26 \mathrm{~cm} \mathrm{H}_{2} \mathrm{O}$. $\mathrm{BMI}>40$ $\mathrm{kg} / \mathrm{m}^{2}$ was the only significant predictive factor (OR 4.35, $\mathrm{p}=0.033$ ) for postrepair shunt placement; 11 of the 15 patients who received shunts had BMI $>40 \mathrm{~kg} / \mathrm{m}^{2}$. Other factors analyzed for significance related to shunt placement are listed in Table 5. Sex did not have a significant effect, nor did leak location or patient age. Prior surgery

TABLE 5. Risk factors associated with shunt placement $(n=15)$

\begin{tabular}{lcc}
\hline \multicolumn{1}{c}{ Variable } & Value $(\%)$ & p Value \\
\hline Age, yrs & & 0.61 \\
\hline$<50$ & $6(40)$ & \\
\hline$>50$ & $9(60)$ & 0.15 \\
\hline Sex & & \\
\hline Male & $1(6.7)$ & \\
\hline Female & $14(93.3)$ & \\
\hline BMI, kg/m ${ }^{2}$ & & \\
\hline$<40$ & $4(26.7)$ & \\
\hline$>40$ & $11(73.3)$ & \\
\hline Leak location & & \\
\hline Cribriform/ethmoid & $9(60)$ & \\
\hline Sphenoid sinus & $4(26.7)$ & \\
\hline Other & $2(13.3)$ & \\
\hline Prior interventions & & $>0.367)$ \\
\hline Surgery for leak repair & $7(46.7)$ & \\
\hline Medical therapy for IIH & $1(6.7)$ & \\
\hline
\end{tabular}

for CSF leak repair was also not correlated with need for shunt placement.

\section{Discussion}

Endoscopic endonasal repair with or without simultaneous craniotomy and postoperative opening pressure (via LP) measurement was an effective treatment in our 46-patient cohort, without significant perioperative morbidity or mortality. The standard management protocol of postoperative LP and opening pressure assessment allows for an identification of underlying $\mathrm{IIH}$ and prompt management of the condition in the same hospitalization, with 12 patients undergoing medical diuretic therapy and 15 patients undergoing CSF diversion. The full protocol and decisionmaking system can be found in flowchart form in Fig. 3. Two of the 46 total patients received revision operations (95.7\% rate of first-repair success), reflecting the durability of the repair, and by utilizing acetazolamide therapy for mild intracranial hypertension, CSF diversion was avoided in 12 of 27 patients with mildly elevated pressure on postoperative LP. The single significant factor associated with shunt placement was morbid obesity (BMI $>40 \mathrm{~kg} /$ $\mathrm{m}^{2}$ ). Overall, this protocol allowed for a reduction in surgical invasiveness, equal if not superior outcomes compared to craniotomy, and efficiency of treatment for the patients.

Regarding patients included in the study who did not receive postoperative LP, $6(50 \%)$ of the 12 had a leak etiology that did not clinically correlate with elevated ICP. Three of these patients had a skull base metastasis that led to their CSF leak after medical therapy, 2 suffered from invasive fungal sinusitis, and 1 had a remote history of skull base trauma, more than 10 years prior to presentation. We believe that these pathologies constitute points on the spectrum of "spontaneous" CSF leaks and should be handled as such for completeness of the medical and surgical treatment of the disease. Once the pathology was identified, these patients were moved into the "non-ICPrelated" arm of our protocol and received pathology-specific treatment thereafter.

Spontaneous CSF rhinorrhea repair has previously shown a $92.8 \%$ success rate via meta-analysis when postoperative management of elevated ICP was performed, versus an $81.8 \%$ success rate when ICP was not managed. ${ }^{14}$ Delineating the etiology of CSF rhinorrhea early in treatment is important to ensure operative repair success. However, preoperative LP may not be the answer, as a patient leaking CSF can have a false-negative normal ICP, or even a "dry tap" and difficulty obtaining CSF. Nor is every spontaneous CSF leak due to IIH: for example, in addition to prior meningitis or skull base fracture, shrinkage of prolactinomas or metastasis to the skull base, leading to nontraumatic spontaneous CSF rhinorrhea, has also been described previously. ${ }^{12}$

The rates of spontaneous CSF leak in the US may be increased up to 2.5-fold in high-obesity regions (e.g., the Midwest), with correlations also found between sleep apnea and hypertension in patients with CSF leak.9 However, the biological mechanism for this increased risk is not entirely clear. Bone mineral density as measured via CT was not significantly different between controls, obese 


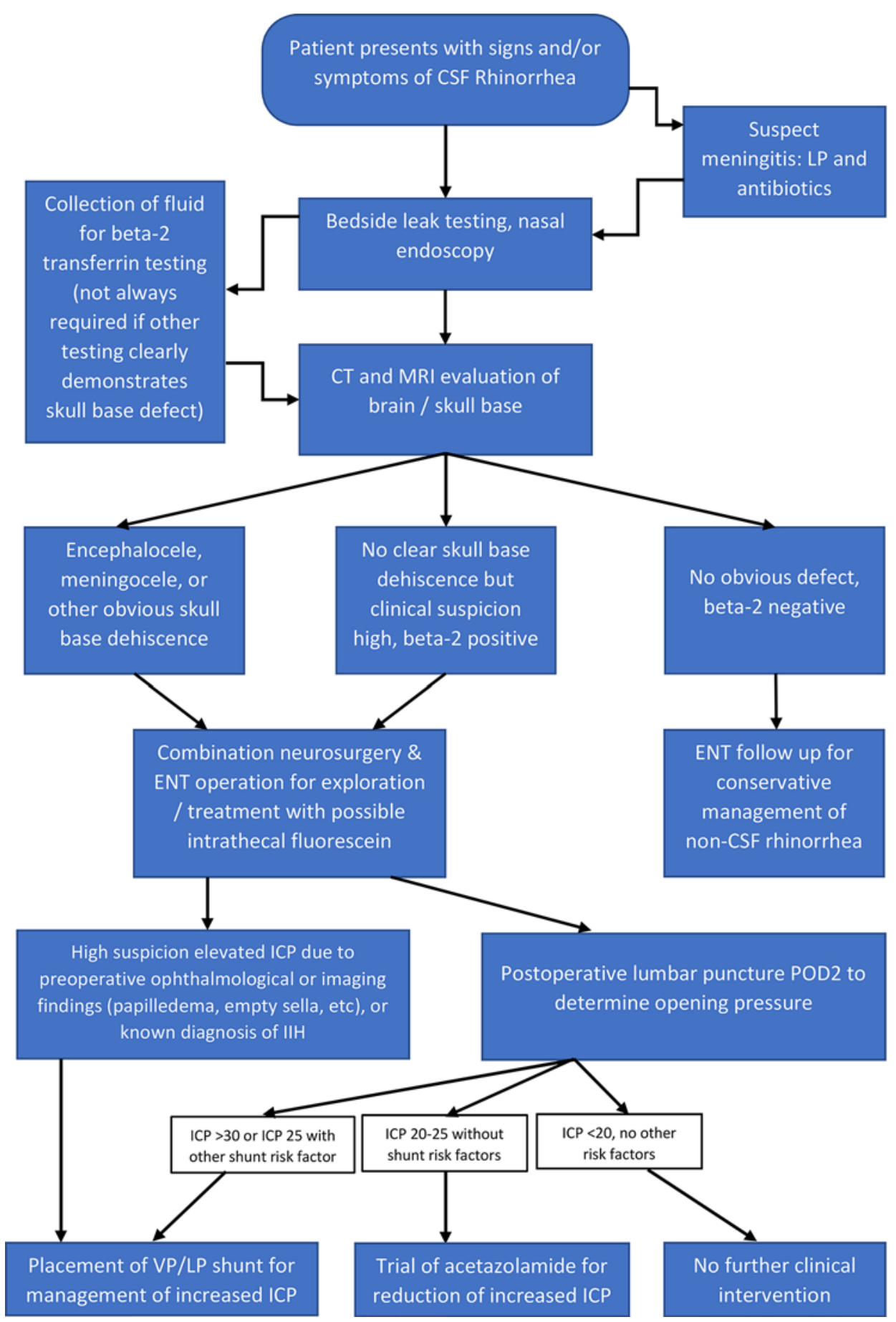

FIG. 3. Decision-making algorithm for patients who present with CSF rhinorrhea. ENT = otolaryngology; LP = lumboperitoneal; POD2 = postoperative day 2; VP = ventriculoperitoneal. Figure is available in color online only.

patients without CSF leak, and patients with CSF leaks.? Furthermore, many patients with obesity and/or IIH never develop CSF leaks. Our hypothesis is that a combination of elevated ICP and congenitally weak areas of the skull base (i.e., cribriform) may lead to leak formation in a subset of patients with IIH; further elucidation of the exact mechanism is warranted in future studies. The key factor in the treatment of spontaneous CSF leaks is the correct identification of the etiology, and an appropriate selection of surgical or medical modalities tailored to the individual patient. The rise in obesity nationwide and worldwide will continue to have a major impact on the incidence of spontaneous CSF leaks due to increased ICP.

There are several limitations to this study. First, while it encompasses 10 years of data and focuses on a relatively rare pathology, the patient cohort is small overall. Drawing 
conclusions from statistical analysis is therefore difficult. Next, the study is retrospective, allowing for inconsistencies in the electronic medical record at the time of initial treatment and therefore in our analysis of cases. A small minority of patients had complicated medical histories, including remote history of trauma, fungal infections, and neoplastic disease, which can all have an effect on clinical outcomes and may cloud the conclusions. Finally, as the surgical management and workup change over time, different patients receive slightly altered versions of the protocol, for example in acetazolamide dosing or when the LP is performed postoperatively, among others.

\section{Conclusions}

Spontaneous CSF leaks are rare and challenging pathologies that often require surgical intervention and are most often due to increased ICP. In this standardized treatment protocol, EEA and postoperative opening pressure measurement yielded a high first-repair success rate with low surgical morbidity and identification of IIH in a significant number of patients. This protocol allows treatment of both the CSF leak and its underlying cause, increasing efficiency of care and reducing the need for revision surgery.

\section{References}

1. Alexander A, Mathew J, Varghese AM, Ganesan S: Endoscopic repair of CSF fistulae: a ten year experience. J Clin Diagn Res 10:MC01-MC04, 2016

2. Bidot S, Levy JM, Saindane AM, Oyesiku NM, Newman NJ, Biousse V: Do most patients with a spontaneous cerebrospinal fluid leak have idiopathic intracranial hypertension? J Neuroophthalmol 39:487-495, 2019

3. Borsetto D, Ciorba A, Cazzador D, Volo T, Denaro L, D'Avellal ED, et al: Transnasal endoscopic management of anterior cerebrospinal fluid (CSF) leak: experience from a large case series. B-ENT 13 (1 Suppl 27):15-21, 2017

4. Conger A, Zhao F, Wang X, Eisenberg A, Griffiths C, Esposito F, et al: Evolution of the graded repair of CSF leaks and skull base defects in endonasal endoscopic tumor surgery: trends in repair failure and meningitis rates in 509 patients. J Neurosurg 130:861-875, 2018

5. D'Anza B, Tien D, Stokken JK, Recinos PF, Woodard TR, Sindwani R: Role of lumbar drains in contemporary endonasal skull base surgery: meta-analysis and systematic review. Am J Rhinol Allergy 30:430-435, 2016

6. Englhard AS, Volgger V, Leunig A, Meßmer CS, Ledderose GJ: Spontaneous nasal cerebrospinal fluid leaks: management of 24 patients over 11 years. Eur Arch Otorhinolaryngol 275:2487-2494, 2018

7. Hatch JL, Schopper H, Boersma IM, Rizk HG, Nguyen SA, Lambert PR, et al: The bone mineral density of the lateral skull base and its relation to obesity and spontaneous cerebrospinal fluid leaks. Otol Neurotol 39:e831-e836, 2018
8. Lobo BC, Baumanis MM, Nelson RF: Surgical repair of spontaneous cerebrospinal fluid (CSF) leaks: a systematic review. Laryngoscope Investig Otolaryngol 2:215-224, 2017

9. Nelson RF, Gantz BJ, Hansen MR: The rising incidence of spontaneous cerebrospinal fluid leaks in the United States and the association with obesity and obstructive sleep apnea. Otol Neurotol 36:476-480, 2015

10. Nix P, Tyagi A, Phillips N: Retrospective analysis of anterior skull base CSF leaks and endoscopic repairs at Leeds. Br J Neurosurg 30:422-426, 2016

11. Nyquist GG, Anand VK, Mehra S, Kacker A, Schwartz TH: Endoscopic endonasal repair of anterior skull base non-traumatic cerebrospinal fluid leaks, meningoceles, and encephaloceles. J Neurosurg 113:961-966, 2010

12. Priddy B, Hardesty DA, Beer-Furlan A, Otto B, Prevedello DM: Cerebrospinal fluid leak rhinorrhea after systemic erlotinib chemotherapy for metastatic lung cancer: a familiar problem from an unfamiliar culprit. World Neurosurg 108:992.e11-992.e14, 2017

13. Rawal RB, Sreenath SB, Ebert CS Jr, Huang BY, Dugar DR, Ewend MG, et al: Endoscopic sinonasal meningoencephalocele repair: a 13-year experience with stratification by defect and reconstruction type. Otolaryngol Head Neck Surg 152:361-368, 2015

14. Teachey W, Grayson J, Cho DY, Riley KO, Woodworth BA: Intervention for elevated intracranial pressure improves success rate after repair of spontaneous cerebrospinal fluid leaks. Laryngoscope 127:2011-2016, 2017

\section{Disclosures}

Dr. Prevedello reports being a consultant for Stryker, Medtronic, and Integra, and being a patent holder for Mizuho, KLS Martin, and ACE Medical.

\section{Author Contributions}

Conception and design: Hardesty, Otto, Carrau, Prevedello. Acquisition of data: Hardesty, Kreatsoulas, Shah. Analysis and interpretation of data: Hardesty, Kreatsoulas. Drafting the article: Hardesty, Kreatsoulas, Shah. Critically revising the article: Hardesty, Kreatsoulas. Reviewed submitted version of manuscript: Hardesty. Approved the final version of the manuscript on behalf of all authors: Hardesty. Statistical analysis: Kreatsoulas, Shah. Administrative/technical/material support: Hardesty, Prevedello. Study supervision: Hardesty.

\section{Correspondence}

Douglas A. Hardesty: Ohio State University Wexner Medical Center, Columbus, OH. douglas.hardesty@osumc.edu. 